\title{
Serial immunogold analysis of foot-and-mouth disease virus (FMDV) non-structural proteins in infected BKLF cells.
} Vivian K. O'Donnell ${ }^{*, * *}$, Christi Cosby, ${ }^{* * * * * * * * *}$, Emiliana Brocchi ${ }^{* * * *}$ and Thomas G. Burrage $^{* * * * * *}$.

"Department of Pathobiology and Veterinary Science, University of Connecticut, Storrs, CT, 61 N. Eagleville Rd., Storrs, CT 06269-3089.

${ }^{* *}$ Foot-and-Mouth Disease Research, Agricultural Research Service, Plum Island Animal Disease Center, P.O. Box 848, Greenport, NY, 11944.

*** Oak Ridge Institute for Science and Education, P.O. Box 117, MS-36, Oak Ridge, TN 37831-0177

***** Instituto Zooprofilattico Sperimental della Lombardia e dell'Emilia Romagna, Brescia, Italy

${ }^{* * * * * *}$ Viral, Cellular and Molecular Imaging, Department of Homeland Security, Plum Island Animal Disease Center, P.O. Box 848, Greenport, NY, 11944.

Picornavirus infection of cells induces a rapid proliferation of cytoplasmic single and double membrane vesicles. These membranes associate into structures called replication complexes (RC) [1-3]. We have been able to visualize mature FMDV particles associated with these membranes [4]. Dual-labeling confocal studies show colocalization of some but not all of FMDV non-structural proteins with structural proteins [4]. In this study, we modified an in situ localization technique with Nanogold ${ }^{\mathrm{TM}}$ [5] to locate non-structural proteins in consecutive sections at $4 \mathrm{hrs}$ pi. Briefly, LFBK cells, were grown on MatTek ${ }^{\mathrm{TM}}$ cover slip dishes, were infected at moi 10 with FMDV O1Campos, were fixed with $4 \%$ paraformaldehyde, permeabilized with $50 \%$ methanol, blocked and incubated with primary and secondary antisera, gold enhanced and processed for EM. The distributions of FMDV's non-structural proteins 2B, 2C, 3C and 3D were determined by incubation with monoclonal and monospecific polyclonal antisera and control antisera followed by anti-species antiserum. A consecutive series of sections shows the organization of the replication complexes and FDMV-associated particles that are visible only after fixation with high concentrations of aldehydes (Figs.1 and 2). Since this treatment interferes with antibody recognition of epitopes and low aldehydes do not preserve particles, we could look only at the membranes of the replication complexes. Antibodies to 2B, 2C and 3D both appear to associate with RC-like membranes (Figs. 3, 4, and 5). Antibody to $3 \mathrm{C}$, on the other hand, is more uniformly distributed in the cytoplasm and there is a consistent localization in the cell nucleus (Fig. 6). Controls using mock infected cells with anti-2B, 2C, 3C and 3D antibodies and infected cells with irrelevant polyclonal and monoclonal antibodies show little background (data not shown). These results indicate that FMDV non-structural proteins 2B, 2C and 3D associate with the $\mathrm{RC}$ and we hypothesize that they could assist in assembly of mature virions. 3C's morphological role, on the other hand, is unclear.

[1] A. Salonen et al., Curr. Top. Microbiol. Immunol. 285 (2005) 139.

[2] D. A. Suhy et al., J. Virol. 74 (2000) 8953.

[3] R. C. Rust et al., J. Virol. 75 (2001) 9808.

[4] V. O. Donnell et al., MSA meeting (2005)

[5] G. Grondin and A. R. Beaudoin. Microsc. Microanal. 7 (2001) 1044. 


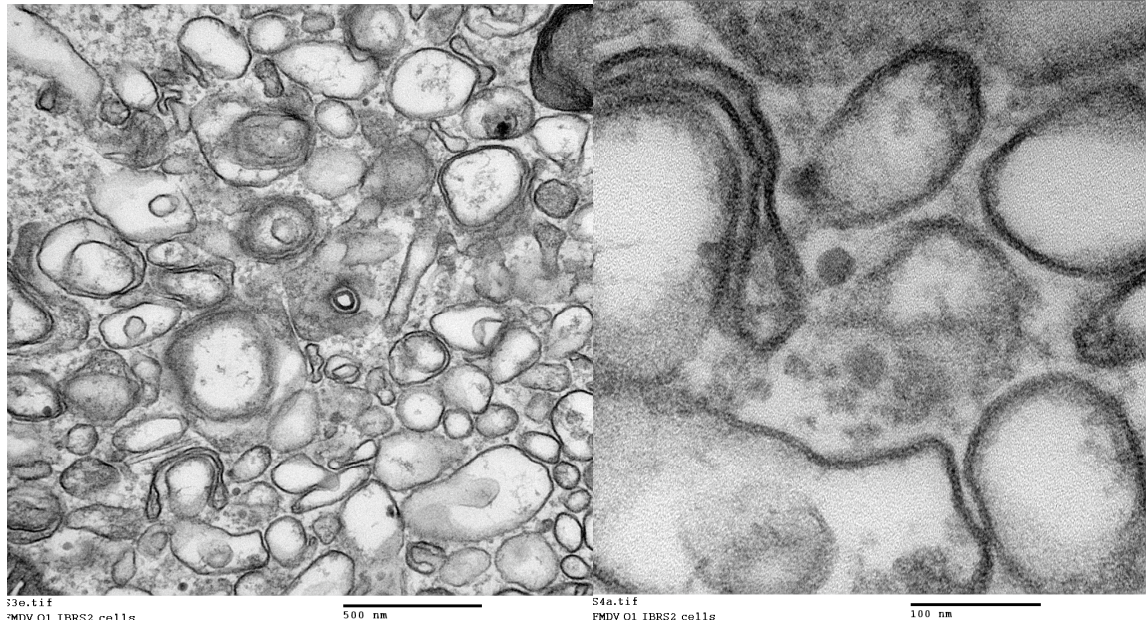

Figures 1 and 2

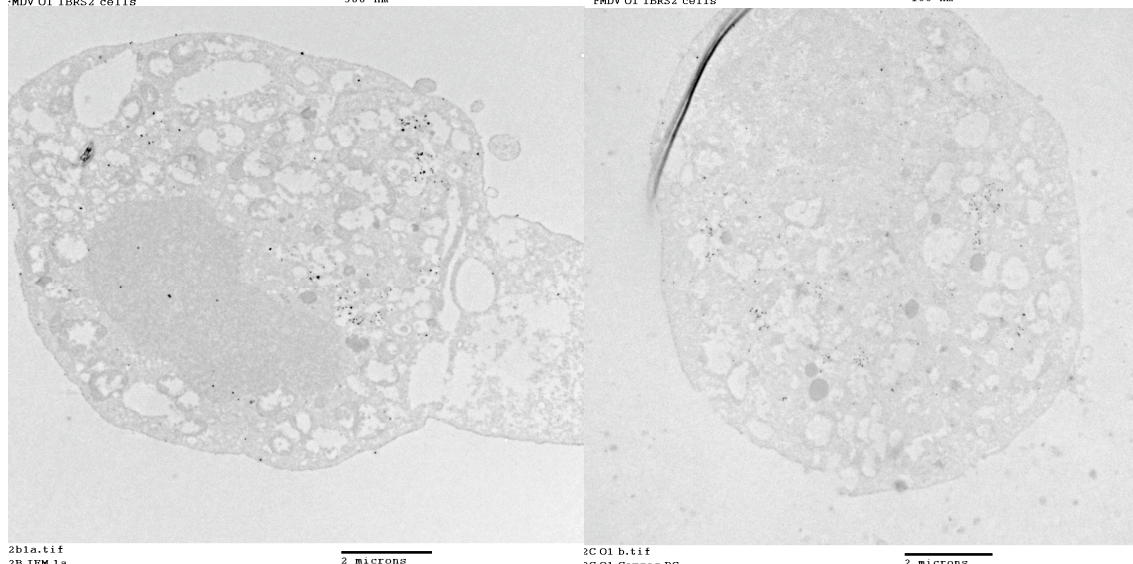

Figures 3

and 4

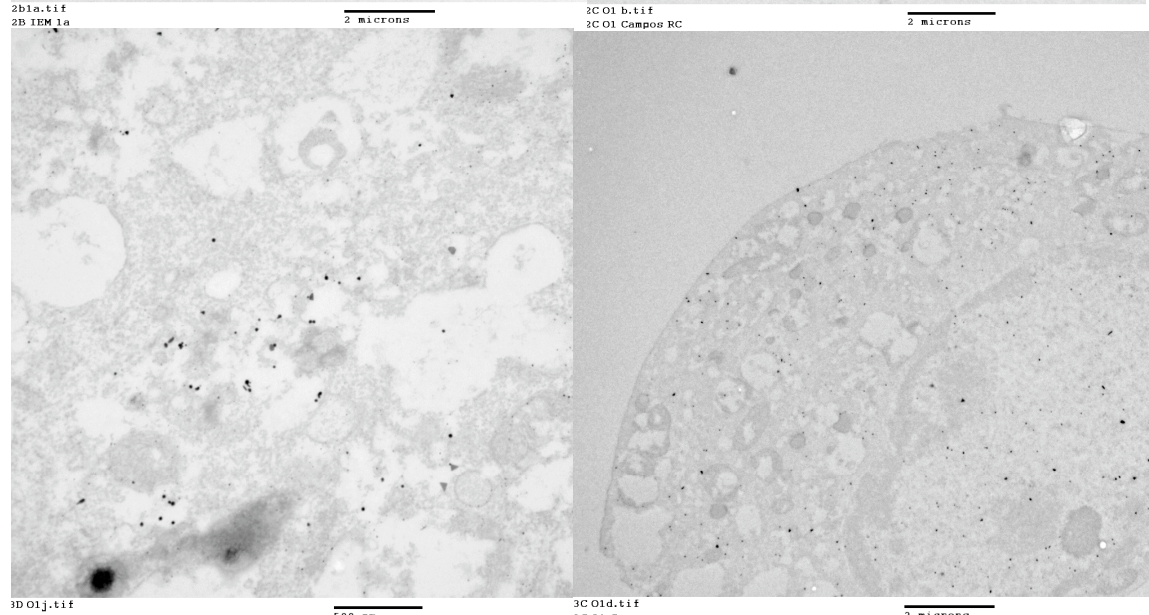

\section{Figures 5} and 6

Figures 1 and 2. Replication complex showing O1 Campos FMDV particles stained on grid with UnAc and Pb Cit. Figures 3-6 not stained. Figure 3. Cell incubated with anti-2B antibody. Figure 4. Section from cell incubated with anti-2C antibody. Figure 5.

Section of cell incubated with anti-3D antibody. Figure 6. Section of cell incubated with anti-3C antibody. 